\title{
PRISMA harms checklist: improving harms reporting in systematic reviews
}

In table 1 of this research methods and reporting paper (BMJ 2016;352:i157, doi:10.1136/bmj.i157), the definitions for adverse event and adverse effect have been switched:

An adverse event should have been defined as "an unfavourable outcome that occurs during or after the use of a drug or other intervention but is not necessarily caused by it." While an adverse effect should have been defined as "an unfavourable outcome that occurs during or after the use of a drug or other intervention and the causal relation between the intervention and the event is at least a reasonable possibility." 